Annals of Pure and Applied Mathematics

Vol. 19, No. 1, 2019, 69-74

ISSN: 2279-087X (P), 2279-0888(online)

Published on 19 February 2019

Annals of

www.researchmathsci.org

DOI: http://dx.doi.org/10.22457/apam.598v19n1a8

Pure and Applied

Mathematics

\title{
Asymptotic Behavior of Solutions of a Singularly Perturbed Differential System of Fractional Order
}

\author{
Burkhan T. Kalimbetov
}

Department of Mathematics, University Akhmed Yasawi, 161200

Turkestan, Kazakhstan.

E-mail: burkhan.kalimbetov@ayu.edu.kz

Received 1 January 2019; accepted 13 February 2019

Abstract. In this paper, we consider the initial problem for systems of differential equations of fractional order with a small parameter for the derivative. Produced regularization problem and is given algorithm for normal and unique solubility general iterative systems of differential equations with partial derivatives.

Keywords: matrix-function, vector-function, differential equation of fractional order, regularization, asymptotics, iterative problems, normal and unique solvability.

AMS Mathematics Subject Classification (2010): 34E10, 34E15

\section{Introduction}

We consider the following singularly perturbed problem

$$
L_{\varepsilon} y(t, \mathcal{E}) \equiv \mathcal{E} y^{(\alpha)}-A(t) y=h(t), \quad y(0, \mathcal{\varepsilon})=y^{0}, \quad t \in[0, T], \quad 0<\alpha<1,
$$

where $y(t, \mathcal{E}) \equiv\left\{y_{1}, \ldots, y_{n}\right\}-$ unknown vector-function, $h(t) \equiv\left\{h_{1}, \ldots, h_{n}\right\}-$ known vectorfunction, $A(t)-n \times n-$ matrix-function, $y^{0}=\left\{y_{1}^{0}, \ldots, y_{n}^{0}\right\}-$ known constant vector, $\varepsilon>0$ - small parameter. It is required to construct a regularized asymptotic solution $[1,2,3,4,5]$ of the problem (1) at for $\varepsilon \rightarrow+0$.

Problem (1) is a Cauchy problem for an ordinary differential equation of fractional order. According to the definition of a fractional order derivative [6,7], i.e. $y^{(\alpha)}(t)=t^{(1-\alpha)} y^{\prime}(t), 0<\alpha<1$, where $y^{\prime}(t)-$ derivative of the first order from the function $y(t)$ by the variable $t$, we write the problem (1) in the following form:

$$
L_{\varepsilon} y(t, \varepsilon) \equiv \varepsilon t^{(1-\alpha)} \frac{d y}{d t}-A(t) y=h(t), \quad y(0, \varepsilon)=y^{0}, \quad t \in[0, T],
$$

We will consider the problem (2) under the following assumptions:

1) matrix-function $A(t)$ and vector-function $h(t)$ belong to the space $C^{\infty}[0, T]$, that is elements of the matrix-function $A(t)$ and components of the vector $h(t)$ have derivatives of any order on the segment $[0, T]$.

2) for any $t \in[0, T]$ the spectrum $\sigma\left\{\lambda_{j}(t)\right\}, j=\overline{1, n}$, of the operator $A(t)$ satisfies the conditions: 


\section{Burkhan T. Kalimbetov}
a) $\lambda_{i}(t) \neq \lambda_{j}(t), i \neq j, \lambda_{j}(t) \neq 0, \quad j=\overline{1, n}$;
b) $\operatorname{Re} \lambda_{j}(t) \leq 0, \quad j=\overline{1, n}, \quad \forall t \in[0, T]$.

\section{Regularization of the problem}

We introduce regularizing variables [2]:

$$
\tau_{j}=\frac{1}{\varepsilon} \int_{0}^{t} s^{(\alpha-1)} \lambda_{j}(s) d s \equiv \varphi_{j}(t, \varepsilon), j=\overline{1, n},
$$

and instead of the problem (2), we will consider «extended» problem

$$
L_{\varepsilon} \tilde{y}(t, \tau, \varepsilon) \equiv \varepsilon t^{(1-\alpha)} \frac{\partial \tilde{y}}{\partial t}+\sum_{j=1}^{n} \lambda_{j}(t) \frac{\partial \tilde{y}}{\partial \tau_{j}}-A(t) \tilde{y}=h(t), \quad \tilde{y}(0,0, \varepsilon)=y^{0} .
$$

Relations of the problem (3) with the problem (2) is that if $\tilde{y}(t, \tau, \varepsilon)$ is a solution of the problem (3), then contraction of the solution

$$
\tilde{y}\left(t, \varphi_{j}(t, \varepsilon), \varepsilon\right) \equiv y(t, \varepsilon)
$$

when $\tau_{j}=\varphi_{j}(t, \varepsilon), j=\overline{1, n}$ will be exact solution of the problem (2).

Defining a solution of the system (3) in the form of series:

$$
\tilde{y}(t, \tau, \mathcal{\varepsilon})=\sum_{k=0}^{\infty} \varepsilon^{k} y_{k}(t, \tau), \quad y_{k}(t, \tau) \in C^{\infty}\left([0, T], C^{n}\right),
$$

we obtain the following iteration problems:

$$
\begin{array}{cc}
L y_{0}(t, \tau) \equiv \sum_{j=1}^{n} \lambda_{j}(t) \frac{\partial y_{0}}{\partial \tau_{j}}-A(t) y_{0}=h(t), & y_{0}(0,0)=y^{0} ; \\
L y_{1}(t, \tau)=-t^{(1-\alpha)} \frac{\partial y_{0}}{\partial t}, & y_{1}(0,0)=0 \\
L y_{k}(t, \tau)=-t^{(1-\alpha)} \frac{\partial y_{k-1}}{\partial t}, \quad y_{k}(0,0)=0, & k \geq 1 .
\end{array}
$$

\section{Solvability of iteration problems}

Solution of each of the iteration problems $\left(\varepsilon^{k}\right)$ will be defined in the space $U$ of functions of the form:

$$
U=\left\{y(t, u): y=y_{0}(t)+\sum_{j=1}^{n} y_{j}(t) e^{\tau_{j}}, y_{j}(t) \in C^{\infty}\left([0, T], C^{n}\right)\right\} .
$$

Each of the iteration problems $\left(\varepsilon^{k}\right)$ has the following form:

$$
L y(t, \varepsilon) \equiv \sum_{j=1}^{n} \lambda_{j}(t) \frac{\partial y_{0}}{\partial \tau_{j}}-A(t) y_{0}=h(t, \tau),
$$

where $h(t, \tau) \in U-$ corresponding right hand side.

The following proposition takes place.

Theorem 1. Let $h(t, \tau) \in U$ and conditions 1) and 2a) hold. Then, for solvability of the equation (6) in space $U$, it is necessary and sufficient that the following conditions hold 
Asymptotic Behavior of Solutions of a Singularly Perturbed Differential System of

$$
\begin{gathered}
\text { Fractional Order } \\
<h(t, \tau), d_{j}(t)>\equiv 0, \quad j=\overline{1, n}, \quad \forall t \in[0, T],
\end{gathered}
$$

where $d_{j}(t)$ - eigenfunctions of the matrix of functions $A^{*}(t)$, corresponding to eigenvalues $\bar{\lambda}_{j}(t), j=\overline{1, n}$.

Proof: Defining a solution $y(t, \tau)$ of the system (6) as an element (5) of the space $U$, we get the following systems for the coefficients $y_{j}(t), j=0,1,2$, of the sum (5):

$$
\begin{aligned}
& {\left[\lambda_{k}(t) I-A(t)\right] y_{k}(t)=h_{k}(t), \quad k=\overline{1, n},} \\
& -A(t) y_{0}(t)=h_{0}(t), \quad(I \equiv \operatorname{diag}(1,1)) .
\end{aligned}
$$

The system (9), due to $\operatorname{det} A(t) \neq 0$, has a unique solution $y_{0}(t)=-A^{-1}(t) h_{0}(t)$.

The system (8) is solvable in $C^{\infty}[0, T]$ if and only if the condition $<h_{k}(t), d_{k}(t)>\equiv 0, k=1,2, \forall t \in[0, T]$, holds, that coincides with the condition (7). Theorem 1 is proved.

Remark 1. If the conditions (7) hold, system (6) has a solution that can be represented as

$$
y(t, \tau)=\sum_{k=1}^{n}\left[\alpha_{k}(t) c_{k}(t)+\sum_{\substack{s \neq k \\ s=1}}^{n} \frac{\left(h_{k}(t), d_{s}(t)\right)}{\lambda_{k}(t)-\lambda_{s}(t)} c_{s}(t)\right] e^{\tau_{k}}-A^{-1}(t) h_{0}(t),
$$

where $\alpha_{k}(t) \in C^{\infty}[0, T], k=\overline{1, n}$ - arbitrary scalar functions.

The following theorem establishes conditions under which the solution (10) of system (6) is uniquely defined in the class $U$.

Theorem 2. Let 1), 2a) hold and $h(t, \tau) \in U$ of the system (6) satisfy conditions (7). Then the system (6) with additional conditions:

$$
\begin{gathered}
y(0,0)=y^{0}, \\
<-t^{(1-\alpha)} \frac{\partial y(t, \tau)}{\partial t}, d_{j}(t)>\equiv 0, \quad j=\overline{1, n}, \quad \forall t \in[0, T],
\end{gathered}
$$

where $y^{0} \in C^{n}-$ known constants, is uniquely solvable in the space $U$.

Proof: Since conditions of Theorem 1 hold, the system (6) has a solution in the space $U$ in the form (10), where functions $\alpha_{k}(t), k=\overline{1, n}$, have not yet been found. To calculate them, we will use additional conditions (11) and (12).

We subject (10) to the initial condition (11), we get the system:

$$
\sum_{k=1}^{n}\left[\alpha_{k}(0) c_{k}(0)+\sum_{s \neq k, s=1}^{n} \frac{\left(h_{k}(0), d_{s}(0)\right)}{\lambda_{k}(0)-\lambda_{s}(0)} c_{s}(0)\right]-A^{-1}(0) h_{0}(0)=y^{0} .
$$

Multiplying scalarly both sides of this equality by $d_{k}(0)$ and taking into account biorthogonality of the systems $\left\{c_{k}(t)\right\}$ and $\left\{d_{k}(t)\right\}$, we uniquely find initial values $\alpha_{k}(0)=\alpha_{k}^{0}$ for the functions $\alpha_{k}(t), k=\overline{1, n}$. 


\section{Burkhan T. Kalimbetov}

We subject now the function (10) to the condition (12). First calculate $\frac{\partial y(t, \tau)}{\partial t}$ :

$$
\sum_{k=1}^{n}\left\{\left(\alpha_{k} c_{k}^{\prime}+\alpha_{k}^{\prime} c_{k}\right)+\left[\sum_{s \neq k, s=1}^{n} \frac{\left(h_{k}, d_{s}\right)^{\prime}\left(\lambda_{k}-\lambda_{s}\right)-\left(h_{k}, d_{s}\right)\left(\lambda_{k}-\lambda_{s}\right)^{\prime}}{\lambda_{k}-\lambda_{s}} c_{s}+\frac{\left(h_{k}, d_{s}\right)}{\lambda_{k}-\lambda_{s}} c_{s}^{\prime}\right]\right\} e^{\tau_{k}}-\left(A^{-1} \cdot h_{0}\right)^{\prime} .
$$

Conditions (12) lead to the equations:

$$
-t^{(1-\alpha)}\left[\alpha_{k}^{\prime}+\left(c_{k}^{\prime}, d_{k}\right) \alpha_{k}+\sum_{\substack{s \neq k \\ s=1}}^{n} \frac{\left(h_{k}, d_{s}\right)}{\lambda_{k}-\lambda_{s}}\left(c_{k}^{\prime}, d_{k}\right)-\left(\left(A^{-1} \cdot h_{0}\right)^{\prime}, d_{k}\right)\right]=0, \quad k=\overline{1, n} .
$$

which together with the initial conditions $\alpha_{k}(0)=\alpha_{k}^{0}$, found earlier, allow us to uniquely find the functions $\alpha_{k}(t), k=\overline{1, n}$. Theorem 2 is proved.

Example 1. Using the algorithm developed above, construct the main term of the asymptotic solution of the Cauchy problem:

$$
\varepsilon\left(\begin{array}{l}
y^{(2 / 3} \\
z^{(2 / 3)}
\end{array}\right)=\left(\begin{array}{cc}
0 & 1 \\
-1 & 0
\end{array}\right)\left(\begin{array}{l}
y \\
z
\end{array}\right)+\left(\begin{array}{l}
h_{1}(t) \\
h_{2}(t)
\end{array}\right), \quad \begin{aligned}
& y(0, \varepsilon)=y^{0}, \\
& z(0, \varepsilon)=z^{0},
\end{aligned}
$$

where $t \in[0, T], T<1, \varepsilon>0-$ small parameter. Eigen values of the matrix $A(t)$ of this system are numbers $\lambda_{1}(t) \equiv-i, \lambda_{2}(t) \equiv+i$. The corresponding eigenvectors $c_{j}(t)$ and eigenvectors $d_{j}(t)$ of the conjugate operator $A^{*}(t)$ have the form:

$$
c_{1}=\left(\begin{array}{c}
-i \\
-1
\end{array}\right), c_{2}=\left(\begin{array}{c}
i \\
-1
\end{array}\right), d_{1}=\left(\begin{array}{l}
i \\
1
\end{array}\right), d_{2}=\left(\begin{array}{c}
-i \\
1
\end{array}\right) .
$$

Introduce regularizing variables:

$$
\tau_{1}=-\frac{3 i}{2 \varepsilon} \sqrt[3]{t^{2}} \equiv \varphi_{1}(t, \varepsilon), \quad \tau_{2}=\frac{3 i}{2 \varepsilon} \sqrt[3]{t^{2}} \equiv \varphi_{2}(t, \varepsilon)
$$

For extended functions $\tilde{w} \equiv\{\tilde{y}(t, \tau, \varepsilon), \tilde{z}(t, \tau, \varepsilon)\}$ we obtain the following problem:

$$
\varepsilon \sqrt[3]{t} \frac{\partial \tilde{w}}{\partial t}+\sum_{j=1}^{2} \lambda_{j} \frac{\partial \tilde{w}}{\partial \tau_{j}}-A \tilde{w}=h(t), \quad \tilde{w}(0,0, \varepsilon)=w^{0},
$$

where $\tilde{w}=\{\tilde{y}, \tilde{z}\}, h(t)=\left\{h_{1}(t), h_{2}(t)\right\}, w^{0}=\left\{y^{0}, z^{0}\right\}$.

Defining a solution of this problem in the form of series

$$
\tilde{w}(t, u, \varepsilon)=\sum_{k=0}^{\infty} \varepsilon^{k} w_{k}(t, u),
$$

we get the following iteration systems:

$$
\begin{aligned}
& L_{0} w_{0}(t, \tau) \equiv \sum_{j=1}^{2} \lambda_{j} \frac{\partial w_{0}}{\partial \tau_{j}}-A w_{0}=h(t), \quad w_{0}(0,0)=w^{0} ; \\
& L_{0} w_{1}(t, \tau)=-\sqrt[3]{t^{2}} \frac{\partial w_{0}}{\partial t}, \quad w_{1}(0,0)=0 ;
\end{aligned}
$$


Asymptotic Behavior of Solutions of a Singularly Perturbed Differential System of Fractional Order

$$
L_{0} w_{k}(t, \tau)=-\sqrt[3]{t^{2}} \frac{\partial w_{k-1}}{\partial t}, \quad w_{k}(0,0)=0, \quad k \geq 1 .
$$

We look for a solution of the equation (14) in the form of the functions:

$$
w_{0}(t, \tau)=w_{1}^{(0)}(t) e^{\tau_{1}}+w_{2}^{(0)}(t) e^{\tau_{2}}+w_{0}^{(0)}(t)
$$

Putting (17) into the equation (14), and equating coefficients at the same exponentials and the free terms, we get:

$$
\begin{aligned}
& {\left[\lambda_{1} I-A\right] w_{1}^{(0)}(t)=0,} \\
& {\left[\lambda_{2} I-A\right] w_{2}^{(0)}(t)=0,} \\
& -A w_{0}^{(0)}(t)=h(t) .
\end{aligned}
$$

From the system (20) we find $w_{0}^{(0)}(t)=-A^{-1} h(t)$. In the equations (18) and (19) $w_{1}^{(0)}(t), w_{2}^{(0)}(t)$-arbitrary functions.

Thus, we have defined solution (17) of the system (14) in the following way:

$$
w_{0}(t, \tau)=\alpha_{1}^{(0)}(t) c_{1} e^{\tau_{1}}+\alpha_{2}^{(0)}(t) c_{2} e^{\tau_{2}}-A^{-1} h(t),
$$

where $\alpha_{k}^{(0)}(t), k=1,2-$ arbitrary functions.

We subject (21) to the initial condition $w_{0}(0,0)=w^{0}$.

$$
\left(\begin{array}{l}
y^{0} \\
z^{0}
\end{array}\right)=\alpha_{1}^{(0)}(0)\left(\begin{array}{l}
-i \\
-1
\end{array}\right)+\alpha_{2}^{(0)}(0)\left(\begin{array}{c}
i \\
-1
\end{array}\right)-\left(\begin{array}{cc}
0 & -1 \\
1 & 0
\end{array}\right)\left(\begin{array}{l}
h_{1}(0) \\
h_{2}(0)
\end{array}\right),
$$

or

then we get:

$$
\left\{\begin{array}{c}
-i \alpha_{1}^{(0)}(0)+i \alpha_{2}^{(0)}(0)+h_{2}(0)=y^{0} \\
-\alpha_{1}^{(0)}(0)-\alpha_{2}^{(0)}(0)-h_{1}(0)=z^{0}
\end{array}\right.
$$

$$
\alpha_{1}^{(0)}(0)=\frac{z^{0}-h_{1}(0)-i\left[h_{2}(0)-y^{0}\right]}{2}, \quad \alpha_{2}^{(0)}(0)=\frac{z^{0}+h_{1}(0)+i\left[h_{2}(0)-y^{0}\right]}{2} .
$$

To uniquely define arbitrary functions $\alpha_{k}^{(0)}(t), k=1,2$, that are present in the solution (21) of the problem (14), we proceed to the next iteration problem (15).

First we calculate:

$$
\frac{\partial w_{0}(t, \tau)}{\partial t}=\dot{\alpha}_{1}^{(0)}(t) c_{1} e^{\tau_{1}}+\dot{\alpha}_{2}^{(0)}(t) c_{2} e^{\tau_{2}}-A^{-1} \dot{h}(t)
$$

Solution of the equation (15) is sought as a function:

$$
w_{1}(t, \tau)=w_{1}^{(1)}(t) e^{\tau_{1}}+w_{2}^{(1)}(t) e^{\tau_{2}}+w_{0}^{(1)}(t) .
$$

Substituting (24) into the equation (15) (taking into account (23)), and equating coefficients at the same exponentials and the free terms, we have:

$$
\begin{gathered}
{\left[\lambda_{1} I-A\right] w_{1}^{(1)}(t)=-\sqrt[3]{t^{2}} \dot{\alpha}_{1}^{(0)}(t),} \\
{\left[\lambda_{2} I-A\right] w_{2}^{(1)}(t)=-\sqrt[3]{t^{2}} \dot{\alpha}_{2}^{(0)}(t),} \\
-A w_{0}^{(1)}(t)=-\sqrt[3]{t^{2}} A^{-1} \dot{h}(t) .
\end{gathered}
$$




\section{Burkhan T. Kalimbetov}

For solvability of the first two systems it is necessary and sufficient that $\dot{\alpha}_{k}^{(0)}(t)=0, k=1,2$. Taking into account the initial conditions (22), we find the functions

$$
\alpha_{1}^{(0)}(t)=\alpha_{1}^{(0)}(0) \equiv \frac{z^{0}-h_{1}(0)-i\left[h_{2}(0)-y^{0}\right]}{2}, \alpha_{2}^{(0)}(t)=\alpha_{2}^{(0)}(0) \equiv \frac{z^{0}+h_{1}(0)+i\left[h_{2}(0)-y^{0}\right]}{2},
$$

unambiguously.

Thus, we defined arbitrary functions $\alpha_{k}^{(0)}(t)=0, k=1,2$, in the solution (21), and thereby, uniquely determined the function (17) of the iteration problem (14), i.e., built the main term of the asymptotics of solutions to the problem (13):

$$
\begin{gathered}
\left(\begin{array}{l}
y_{\varepsilon 0}(t) \\
z_{\varepsilon 0}(t)
\end{array}\right)=\left[\frac{z^{0}-h_{1}(0)-i\left(h_{2}(0)-y^{0}\right)}{2}\right]\left(\begin{array}{c}
-i \\
-1
\end{array}\right) e^{-\frac{3 i}{2 \varepsilon} \sqrt[3]{t^{2}}}+ \\
+\left[\frac{z^{0}+h_{1}(0)+i\left(h_{2}(0)-y^{0}\right)}{2}\right]\left(\begin{array}{c}
i \\
-1
\end{array}\right) e^{\frac{3 i}{2 \varepsilon} \sqrt[3]{t^{2}}}-\left(\begin{array}{cc}
0 & -1 \\
1 & 0
\end{array}\right)\left(\begin{array}{l}
h_{1}(t) \\
h_{2}(t)
\end{array}\right) .
\end{gathered}
$$

Acknowledgments. This work is supported by the grant AP05133858 "Contrast structures in singularly perturbed equations and their application in the theory of phase transitions" by the Scientific Committee of the Ministry of Education and Science of the Republic of Kazakhstan. Also, I am grateful to the reviewer for the suggestion to improvement of the paper.

\section{REFERENCES}

1. S.A.Lomov, Introduction to General Theory of Singular Perturbations. Vol. 112 of Translations of Mathematical Monographs, American Mathematical Society, Providence, USA (1992).

2. B.Kalimbetov and V.Safonov, A regularization method for systems with unstable spectral value of the kernel of the integral operator, Journal of Differential Equations, 31 (1995) 647-656.

3. B.Kalimbetov, M.Temirbekov and Z.Khabibullayev, Asysmptotic solutions of singular perturbed problems with an instable spectrum of the limiting operator, Journal of Abstract and Applied Analysis, Article no. 120192 (2012).

4. B.Kalimbetov, Regularized asymptotics of solution for systems of singularity perturbed differential equations of fractional order, Inter. J. Fuzzy Math. Archive, 16(1) (2018) 67-74.

5. B.Kalimbetov, On the Question of Asymptotic Integration of Singularly Perturbed Fractional-Order Problems, Asian J. of Fuzzy and Appl. Math., 6(3) (2018) 44-49.

6. U. Katugampola, Correction to "What is a fractional derivative?" by Ortigueira and Machado, Journal of Computational Physics, 293 (2015) 4-13.

7. R. Khalil, M. Al Horani, A. Yousef and M. Sababheh, A new definition of fractional derivative, Journal Comput. Appl. Math., 264 (2014) 65-70. 